\title{
Phage therapy: a potential alternative in the treatment of multi-drug resistant bacterial infections
}

\begin{abstract}
The development of antibiotic-resistant in bacteria has enhanced the scope of developing alternative approaches to conventional drugs. Bacteriophage therapy is one such approach that can be used as an alternative to antibiotics. The significance of live phages in counteracting lethal infectious and life threatening diseases caused by Gram positive and Gram negative bacteria has been reported. Bacteriophage therapy is a significant alternative to antibiotics in the current era of multidrug resistant bacterial infections as phages are used topically, orally or systemically in studies reviewed. The success rates found in these studies were $80-95 \%$ with few gastrointestinal or allergic side effects. British studies also verified significant effectiveness of phages against Escherichia coli, Acinetobacter spp., Pseudomonas spp and Staphylococcus aureus. US studies dealt with improving the bioavailability of phage. Problems faced in these studies have also been discussed. Conclusively, phage therapy may prove as an important alternative to antibiotics for combating multidrug resistant pathogens.
\end{abstract}

Volume 5 Issue 7 - 2017

\author{
Adebayo OS, I Gabriel Ajobiewe RAO,2 \\ Taiwo MO,I Kayode JSI \\ 'Department of Microbiology, Federal University of Agriculture, \\ Nigeria \\ 2Federal University of Oye-Ekiti, Ekiti State, Nigeria
}

Correspondence: Adebayo OS, Department of Microbiology, Federal University of Agriculture, Abeokuta, Ogun State, P.M.B:2240, Nigeria, Tel 2347030303230,

Email iamoluwatobi@yahoo.com

Received: September 21, 2017 | Published: November 15, 2017

Keywords: Bacteriophage, Infectious disease, Drug resistance

\section{Introduction}

Phages are viruses that infect the bacteria and causes bacterial cell lysis. ${ }^{1}$ They are "programmed" to terminate one or a few kinds of different strains of bacteria. Phages are widespread in nature and can appear naturally in food and in the human body (for example in the intestines). Phages can efficiently destroy bacteria which have built up resistance over time to antibiotics and which causes life-threatening infections. ${ }^{1,2}$ Phage therapy was introduced by Felix d'Herelle, who co discovered phages around $1920 .{ }^{3}$ These findings occurred about 20 years prior to practical application of penicillin, the first antibiotic. At the time of its discovery, phage therapy was being considered as a potential treatment technique against bacterial infectious diseases. Phage therapy was used to treat and prevent infectious bacterial diseases in the former Soviet Union and Eastern Europe and was abandoned by the West in the 1940s with antibiotic era springing up.

Bacteriophages are regarded as one of the possible alternatives to antibiotics in animal therapy, prophylaxis and reduction of bacterial loads in food products of animal origin. Trials with bacteriophages and farm animals have been proven to be successful and thereby used to destroy food borne pathogen such as Escherichia coli, ${ }^{4}$ Salmonella $^{4}$ and Campylobacter. ${ }^{5}$

\section{Life cycles of phages}

Phages possess a developmental cycle inside the host bacteria which can be lytic or lysogenic. ${ }^{6}$ The lytic cycle covers a series of events that occur between attachment of phage particle to a bacterial cell and its subsequent release of new daughter phage particles. It consists of five stages, namely; adsorption of phage to host cell, penetration of phage nucleic acid, transcription and translation, assembly and exit.

The lysogenic cycle on the other hand involves replication of phage nucleic acid together with the host genes for numerous generations without major metabolic consequences for the cell. ${ }^{6}$ This is known as a latent mode of infection which occurs at a very low frequency. The phage genes in this state may occasionally return to lytic cycle, leading to release of phages particles. This phenomenon is known as lysogeny and phages that can develop both lytically and lysogenically are regarded as temperate phages. ${ }^{6}$

\section{Phage therapy}

Phage therapy combats bacterial infections of humans (or animals) with the goal of reducing bacterial load. Findings shows that appropriate administration can be used to treat lethal infectious diseases caused by Escherichia coli, Vibro cholerae, Staphylococcus aureus and Salmonella spp. ${ }^{7}$ Phages can be delivered topically, orally, directly into body tissues, or systemically. A second means in which therapies differ is in terms of delivery. Phage - infected bacteria may be employed as a means of delivering phages to intracellular pathogens. ${ }^{8}$ Finally, the normal ability of lytic phages to destroy infected bacteria may be exploited, as is generally the case with phage therapy, or alternatively phages may also be engineered to deliver non-phage genes coding for antibacterial agents. ${ }^{9}$

The success of phage therapy is a function of elementary principles of phage-bacterial infection. One must first recognize a phage that can infect a bacterium, and the effectiveness relies on the extent of phage bacterial infection. Secondly, if treatment is initiated during early stages of infections it may be essential to compensate, by using a larger phage dosage, due to the inability of too-few bacteria to successfully expand phage population. ${ }^{10}$ The advent of antibiotic resistance bacteria has grown into a major problem for clinician over the last 2-3 decades. In United State, antibiotic resistance bacteria infect approximately 2 million people each year of which at least 23,000 people has been documented to die annually. ${ }^{11}$ In recent times, bacteria such as Acinetobacter baumannii, Staphylococcus aureus, Pseudomonas aeruginosa, Enterococus faecium, Klebsiella pneumonia have developed multi-drug resistance and cause major healthcare disaster in ICUs. ${ }^{12,13}$

The development of severe infectious diseases resistant to antibiotics has been an issue of serious concern and this in turn has led to the discovery of alternative approaches that could replace these 
conventional antibiotics. Steady decline in the discovery of new class of antibiotics, has enhanced the scope of bacteriophage as an alternative to conventional antimicrobial drugs. Therefore, bacteriophage can be employed as therapeutic agent to combat infections caused by multidrug resistance bacteria. ${ }^{14,15}$

\section{Clinical studies of phages in human patients}

In human, a number of infectious diseases such as septicemia, wound infections, skin infection, osteomyelitis, urinary tract infections and middle ear infections were effectively treated with phage therapy. ${ }^{16}$ In the US, phages were commonly used for preparation of human and animal vaccine. In phage therapy, phages were used in the form of liquid suspension, tablets, creams rinses, aerosols. ${ }^{17,18}$ Phages therapy trials have been carried out in some countries and it is discussed below.

\section{Polish studies}

Slopek et al. ${ }^{19}$ reported cases of the treatment of staphylococcal infected patients of which 151 cases were of mixed infection including Staphylococcus. Positive results were obtained in $75 \%$ of infected ulcerated varicose vein cases and $100 \%$ in gastrointestinal infection, pericarditis and furunculosis due to Staphylococcus. Response rates were lower for patients more than 60 years of age and in patients with mixed infections. Interestingly, parallel administration of antibiotics diminished the effectiveness of phage therapy from $95.2 \%$ to $84.9 \%$.

Weber et al. ${ }^{20}$ evaluated the extent to which orally administered anti staphylococcal and anti-pseudomonal phage were penetrated into the serum or urine of 56 patients with suppurative bacterial infection. By the tenth day of treatment, it was established that $84 \%$ serum samples and 35\% urine samples contained phage indicating a high bio-availability ${ }^{21}$

\section{British studies}

Soothill 22 verified the ability of phages in experimental Acinetobacter, Pseudomonas and staphylococcal infection in mice and the research reported limited use of phage therapy in staphylococcal infections. Soothill then shifted attention to the problematic cases of cutaneous infection due to burns among patients with graft failure leading to bacterial infection. The study was experimented in guinea pigs and split skin grafts were taken and full thickness defects were created. ${ }^{23}$ Prior to re implantation of graft, wound bed was infected with Pseudomonas strain with its respective phage. After five days, Grafts were accepted and they vascularized. ${ }^{23}$

\section{US studies}

Merrill et al. ${ }^{24}$ discovered a system to overcome this sequestration of phage in the reticulo endothelial system. He injected $\lambda$ phage into mice and isolated the phage. This phage was re grown in a mutant $E$. coli strain repeatedly ten times. This serial cycling of phage proved effective in selecting $\lambda$ with an amino acid mutation in major phage head protein that enabled it to evade thereticulo endothelial system. ${ }^{24}$

\section{Phage therapy in animals}

Bacteriophages are broadly used for the treatment of infections caused by Escherichia coli and Salmonella in chickens, calves, pigs and lambs. Smith and Huggins ${ }^{25}$ experimentally proved that, a single dose of phage $\mathrm{R}$ is effective in averting the mortality in mice due to septicemia caused by E.coli which cannot be treated with multiple doses of antibiotics. They consequently used bacteriophages for the treatment of infections caused by entero toxigenic E.coli in neonatal lambs, calves and pigs. ${ }^{25}$ Vintage research on the therapeutic ability of phages were conducted by Felix d'Herelle, who carried out field studies on fowl typhoid (Salmonella enterica subspecies enterica serovar Gallinarum) and laboratory studies on dysentery in rabbits (Shigella dysenteriae). ${ }^{3}$ Successive animal studies have revealed that phage therapy is effective in animal systems. René Dubos et al. ${ }^{26}$ at Harvard University (USA) showed that anti-S dysenteriae phage injected intraperitoneally in mice showed in the blood stream (and even crossed the brain-blood barrier) but were rapidly cleared by the reticuloendothetial system (particularly the spleen).

\section{Advantages of phage therapy over antibiotics}

Advantages of phage therapy over the use of chemical antibiotics can be framed in terms of phage properties.

\section{Bactericidal agents}

Bacteria that have been successfully infected by obligate lytic phages are unable to regain their viability. In contrast, certain antibiotics such as tetracycline are bacteriostatic, and result in bacterial resistance. ${ }^{27}$

\section{Auto "dosing"}

Phages during bacterial-lysing process have the capability of increasing in numbers specifically where the hosts are located, though with constraint such as dependence on relatively high bacterial densities. ${ }^{28}$ This is known as Auto "dosing" because the phages themselves backs the establishment of the phage dose. ${ }^{28}$

\section{Minimal disruption of normal flora}

Due to their host specificity- This ranges from the ability to infect few strains of bacterial species to more than one relatively closely related bacterial genus. However, numerous conventional antibiotics, which have broader spectrums of activity, are predisposed to induce infections, such as antibiotic associated Clostridium difficile colitis or Candida albicans yeast infections. The preference towards developing only broader spectrum antibiotics, however, may be changing. ${ }^{29}$

\section{Rapid discovery}

Phages acting against many pathogenic bacteria are found in sewage and other waste materials that contain high bacterial concentrations. However, the host bacteria are difficult to culture and may differ based on the number of phage types affecting them. ${ }^{30}$ Unlike antibiotics, which can be toxic, phages that display little or no toxicity can be isolated against most target bacteria.

\section{Biofilm clearance}

Biofilms are complex in nature and can resist a wide range of antibiotics when compare to planktonic bacteria. Phages however have a proven ability to clear at least some biofilms, possibly due to their ability to infiltrate their way into biofilms by lysing a bacterial layer at a time, or owing to the display of biofilm exopolymerdegrading depolymerases. ${ }^{31}$

\section{Narrower potential for inducing resistance}

The narrow host range demonstrated by most phages restricts the number of bacterial types for specific phage-resistance mechanisms. There are contrasts with the large fraction of bacteria that can be affected by most conventional antibiotics. ${ }^{27}$ This implies that when patients are infected with multiple bacteria, in this situation bacteriophage therapy will not effective. 


\section{Shortcomings of phage therapy}

\section{Not all phages make good therapeutics}

Good therapeutic phages should possess a high potential to reach and kill the bacteria in combination with a low potential to otherwise negatively modify the environments to which they are applied. The usage of temperate phages as therapeutics is challenging owing to a combination of display of super infection immunity, ${ }^{32}$ which then converts phage-sensitive bacteria into insensitive ones, and the encoding of bacterial virulence factors, including bacteria toxins.

In order to avoid temperate or toxin-carrying phages, the phages displaying poor lysing potential against target bacteria are excluded. Such low "virulence" can be due to poor adsorption properties, low potential to evade bacterial defenses, or poor replication characteristics. Ideally, phages should also parade low potential to exchange bacterial genes between bacteria. ${ }^{30}$

\section{The problem of narrow host range}

No antimicrobials displaying selective toxicity will have effect on all possible microbial targets. Usually the narrowness of phage host ranges- a few strains, a few species, or much rarer, a few genera of bacteria, will at the lowest place limits on presumptive treatment, i.e., treatment courses that begin earlier to the identification of the pathogen's susceptibility to antibacterial such as to specific phages. ${ }^{32}$ However, as phages can often be employed in combination with other antibacterial agents, including other phages (so-called phage cocktails), the lytic range of phage products can often be much broader than the range of activity of individual phage types. ${ }^{32}$

\section{Conclusion}

It's quite obvious from the above study that the use of bacteriophages to control bacterial infections shows therapeutic promise. The worldwide increase of pathogenic bacteria resistant to antibiotics makes it imperative to exploit alternative strategies to combat this threat. The therapeutic use of bacteriophages - perhaps in combination with antibiotics - may turn out to be a valuable approach. However, it is also quite clear that the safe and controlled use of phage therapy will require detailed information on the properties and behavior of the specific phage-bacterium system, both in vitro and in vivo. The in vivo susceptibility of bacterial pathogens to bacteriophages is poorly understood.

Phages, as antibacterial agents, have a number of properties that make them alternatives to chemical antibiotics while most or perhaps all concerns associated with phage therapy should be manageable through a combination of proper phage selection, effective formulation, and greater clinician understanding of and familiarity with product application. Use of antibiotics along with phages has been tried with limited benefit because antibiotics are more likely to inhibit phage effectiveness than to enhance it and combination may result in problem/resistant genes. Hence it is concluded that phage therapy alone is more effective.

In medicine in recent times, phages find many applications. They are used for typing of clinical bacterial strains for in situ bacterial detection through labeled phages, (Listeria), phage display system for vaccines, control of food pathogens and for drug and gene delivery using defective phage with targeted receptor. Phage therapy for eliminating multidrug resistant bacteria is gaining importance. Nevertheless, more studies are still required before phages can be considered a real approach for therapy of bacterial infections. The usage of phages for therapeutic purposes has need of specific phages for the pathogenic strains of a precise environmen. ${ }^{33}$

\section{Acknowledgments}

None.

\section{Conflicts of interest}

There is no conflict of interest.

\section{References}

1. Biswas B, Adhya S, Washart P, et al. Bacteriophage therapy rescues mice bacteremic from a clinical isolate of vancomycin- resistant Enterococcus faecium. Infect Immun. 2002;70(1):204-210.

2. Wang $\mathrm{J}, \mathrm{Hu} \mathrm{B}, \mathrm{Hu} \mathrm{J}$, et al. Use of bacteriophage in the treatment of experimental animal bacteremia from imipenem-resistant Pseudomonas aeruginosa. Int J Mol Med. 2006;17(2):309-317.

3. Summers WC. Bacteriophage therapy. Annual Rev Microbiol 2001;55:437-451.

4. Huff WE, Huff GR, Rath NC. Evaluation of the influence of bacteriophage titer on the treatment of Colibacillosis in broiler chickens. Poult Sci. 2016;85(8):1373-1377.

5. Loc Carrillo C, Atterbury RJ, Connerton IF. Bacteriophage therapy to reduce Campylobacter jejuni colonization of broiler chickens. Appl Environ Microbiol. 2005;71(11):6554-6563.

6. Benett PM. How TGB Bacterial and bacteriophage genetics. (9th edn), 1998;2:231-286.

7. Matsuzaki S1, Yasuda M, Nishikawa H, et al. Experimental protection of mice against lethal Staphylococcus aureus infection by novel bacteriophage fMR11. J Infect Dis. 2003;187(4):613-624.

8. Broxmeyer L, Sosnowska D, Miltner E, et al. Killing of Mycobacterium avium andMycobacterium tuberculosisby mycobacteriophagedelivered by a non-virulent mycobacterium. $J$ infect dis. 2002;186(8):1155-1160.

9. Westwater C, Kasman LM, Schofield DA, et al. Use of genetically engineered phage to deliver antimicrobial agents to bacteria:an alternative therapy for treatment of bacterial infections. Antimicrob agents Chemother. 2003;47:1301-1307.

10. Kasman LM, Kasman A, Westwater C, et al. Overcoming the phage replication threshold:a mathematical model with implications for phage therapy. J Virol. 2002;76(11):5557-5564.

11. CDC. Antibiotic resistance threats in the United States. Centre of Diseases Control and Prevention, USA. 2013.

12. Blair JMA, Webber MA, Baylay AJ, et al. Molecular mechanisms of antibiotic resistance. Nat Rev Microbiol. 2015;13(2):42-51.

13. Wittebole X, De Roock S, Opal SM. A historical overview of bacteriophage therapy as an alternative to antibiotics for the treatment of bacterial pathogens. Virulence. 2014;5(1):226-235.

14. Lu TK, Koeris MS. The next generation of bacteriophage therapy. Curr Opinion Microbiol. 2011;14(5):524-531.

15. Gill JJ, Hyman P. Phage choice, isolation and preparation for phage therapy. Curr Pharm Biotechnol. 2010;11(1):2-14.

16. Kutter E, Vos D, Gvasalia G, et al. Phage therapy in clinical practice:Treatment of human infections. Curr Pharma Biotechnol. 2012;11(1):69-86

17. Qadir MI. Phage therapy:A modern tool to control bacterial infections. Pak J Pharm Sci. 2015;28(1):265-270.

18. Kutateladze M, Adamia R. Bacteriophages as potential new therapeutics to replace or supplement antibiotics. Trends Biotechnol. 2010;28(12):591-595. 
19. Slopek S, Weber-Dabrowska B, Dabrowski M, et al. Results of bacteriophage treatment of suppurative bacterial infections in the years 1981-1986. Arch Immunol Ther Exp. 1987;35(5):569-583.

20. Weber-Dabrowska B, Mulczyk M, Górski A. Bacteriophage therapy for infections in cancer patients. Clin Appl Immunol Rev. 2001;1(3-4):131-134.

21. Alisky J, Iczkowski K, Rapoport A, et al. Bacteriophages show promise as antimicrobial agents. $J$ Infect. 1998;36(1):5-15.

22. Soothill JS. Treatment of experimental infections of mice with bacteriophages. J Med Microbiol. 1992;37(4):258-261.

23. Soothill JS. Bacteriophage prevents destruction of skin grafts by Pseudomonas aeruginosa. Burns. 1994;20(3):209-211.

24. Merril CR, Biswas B, Carlton R. Long-circulating bacteriophage as antibacterial agents. Proc Natl Acad Sci USA. 1996;93(8):3188-3192.

25. Smith HW, Huggins MB. Effectiveness of phages in treating experimental Escherichia coli diarrhea in calves, piglets and lambs. $J$ Gen Microbiol. 1993;129(8):2659-2675.

26. Dubos RJ, Straus JH, Pierce C. The multiplication of bacteriophage in vivoand its protective effect against an experimental infection with Shigella dysenteriae. J Exp Med. 1943;78(3):161-168.
27. Carlton RM. Phage therapy:past history and future prospects. Arch Immunol Ther Exp. 1999;47(5):267-274.

28. Abedon ST, Thomas-Abedon C. Phage therapy pharmacology. Curr Pharm Biotechnol. 2010;11(1):28-47.

29. Fischbach MA, Walsh CT. Antibiotics for emerging pathogens. Science. 2009;325(5944):1089-1093.

30. Krylov VN. Phagotherapy in terms of bacteriophage genetics:hopes, perspectives, safety, limitations. Genetika. 2001;37(3):869-887.

31. Abedon ST. Kinetics of phage-mediated biocontrol of bacteria. Foodborne Pathog Dis. 2009;6(7):807-815.

32. Hyman $\mathrm{P}, \mathrm{Abedon} \mathrm{ST}$. Bacteriophage host range and bacterial resistance. Adv Appl Microbiol. 2010;70:217-248.

33. O'Flynn G, Coffey A, Fitzgerald GF. The newly isolated lytic bacteriophages st104a and st104b are highly virulent against Salmonella enterica. J Appl Microbiol. 2006;101(1):251-259. 